\title{
Was bessert Gesichtsfelddefekte nach Schlaganfall?
}

\author{
Welche Rolle spielt das Training \\ der räumlichen Aufmerksamkeit \\ bei der Behandlung von Gesichts- \\ felddefekten nach Schlaganfall?
}

- In der Rehabilitation von Gesichtsfelddefekten hat sich das visuelle Explorationstraining in den letzten Jahren zunehmend etabliert. Augenbewegungen werden mit dem Ziel trainiert, das vorhandene Sehfeld effektiver abzusuchen und so den Gesichtsfelddefekt teilweise zu kompensieren. Das Training beruht wesentlich auf visuellräumlicher Aufmerksamkeit. Daher ist unklar, ob die in bisherigen Studien veröffentlichten Verbesserungen bei explorativen Augenbewegungen und Alltagsaktivitäten nicht wesentlich auf eine Verbesserung der räumlichen Aufmerksamkeit zurückgehen.

46 konsekutive Patienten mit postchiasmatischen Läsionen (überwiegend ischämische Infarkte) und seit mindestens drei Monaten bestehenden Gesichtsfelddefekten erhielten entweder ein visuelles Explorationstraining (Gruppe A) oder zunächst nur ein visuell-räumliches Aufmerksamkeitstraining und anschließendes Explorationstraining (Gruppe B). Gesichtsfelder, Augenbewegungen, Lesen und andere Alltagsaktivitäten wurden vor und nach

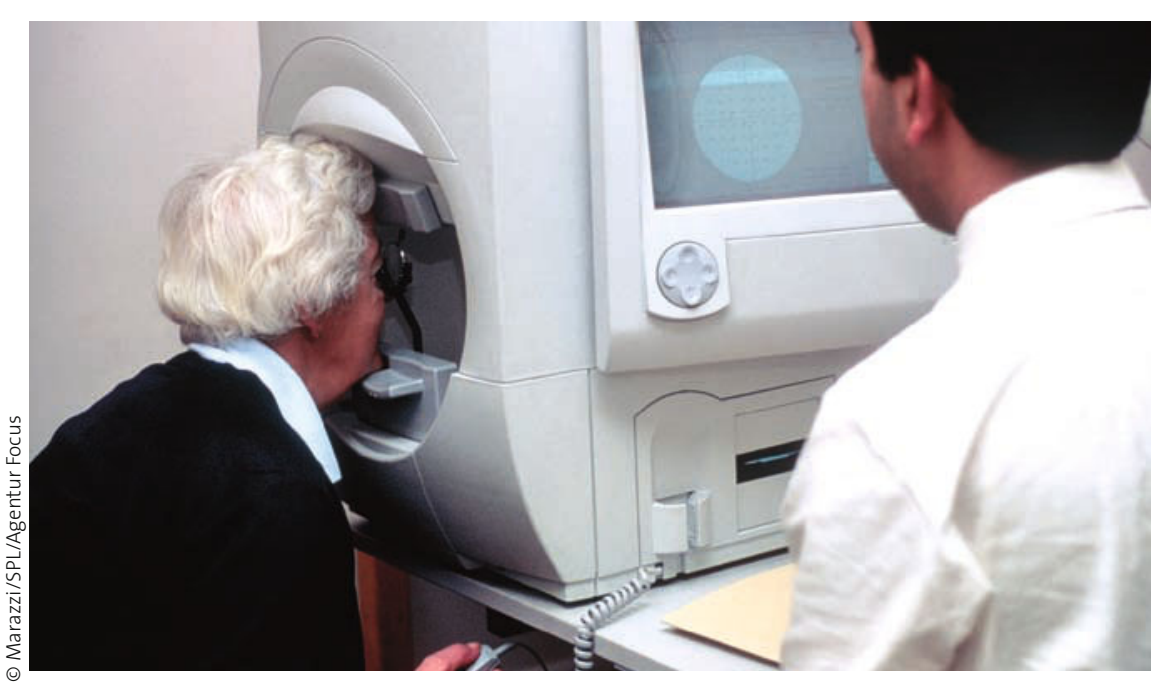

Gesichtsfelduntersuchung: Kann Aufmerksamkeitstraining Defekte verbessern?

jeder Intervention gemessen. In der Gruppe B wurden die Zielparameter vor und nach dem Aufmerksamkeitstraining und nach dem Explorationstraining gemessen.

Bei nahezu allen Zielparametern wurden Verbesserungen in beiden Gruppen beobachtet. Dabei unterschieden sich der Umfang der Verbesserungen beim Explorationstraining und beim Aufmerksamkeitstraining nicht voneinander. Nur beim Lesen wurde bei keiner der beiden Interventionen eine signifikante Verbesserung erzielt.
Allein mit Aufmerksamkeitstraining können vergleichbare Verbesserungen in Bezug auf explorative Augenbewegungen und Alltagsaktivitäten erreicht werden wie mit visuellem Explorationstraining. Die Aufmerksamkeit hat eine große Bedeutung für Verbesserungen, die durch visuelles Explorationstraining erreicht werden.

- A. R. Lane et al.

Visual exploration training is no better than attention training for treating hemianopia. Brain 133 (2010) 1717-1728

\section{Kommentar}

Diese Arbeit versucht erstmals, die aktiven Bestandteile des Explorationstrainings bei Gesichtsfelddefekten näher zu beschreiben. Der Ansatz, dieses Vorgehen mit Aufmerksamkeitstraining zu vergleichen, ist originell und gleichsam ambitioniert. Methodisch hat die Arbeit einige Schwächen, welche die Interpretation der Daten erschweren: Das Trainingsfeld bei dem hier verwendeten visuellen Explorationstraining auf einem Laptopmonitor war klein ( $30^{\circ}$ horizontal, $15^{\circ}$ vertikal). Die Interventionen wurden nicht mit Übungstherapien wie Krankengymnastik und Ergotherapie gekoppelt. Es erfolgte keine randomisierte Zuordnung zur In- terventionsgruppe. Die Auswertung der Messparameter erfolgte nicht verblindet. Die Stichprobengröße ist für eine Interventionsstudie klein. Ohne vorherige Berechnung des Stichprobenumfanges besteht bei kleiner Fallzahl die Möglichkeit, dass die Nullhypothese fälschlicherweise angenommen wurde.

Dennoch ist es den Autoren gelungen, mit dem von ihnen entwickelten Protokoll die Rolle der visuell-räumlichen Aufmerksamkeit bei der Rehabilitation von Gesichtsfelddefekten besser zu charakterisieren. Zweifellos erfordert diese wichtige Frage eine weiterführende, vor allem größere Studie.

\section{Nicht teilen kann man die Auffassung} der Autoren in ihrer Diskussion, das Aufmerksamkeitstraining könne zu Hause (ohne therapeutische Kontrolle?) auf handelsüblichen elektronischen Geräten wie Mobiltelefonen oder portablen PCSpiele-Konsolen durchgeführt werden. Man muss starke Zweifel haben, dass die Zielgruppe der von Gesichtsfelddefekten Betroffenen und überwiegend über 70-Jährigen mit erheblichen Sehbehinderungen in der Lage ist, kleine elektronische Kommunikationsgeräte zu therapeutischen Zwecken sinnvoll einsetzen zu können.

G. Nelles — 